\title{
IgA1 from HSP Patients Trigger Apoptosis and Inhibit Cytoskeletal Proteins in HUVEC
}

\author{
Liping Yuan ${ }^{*}$, Wenjun Fei², Lin Wu${ }^{1}$, Ming Gui' ${ }^{1}$, Qin Zhang1, Bo $\mathrm{Hu}^{1}$ \\ ${ }^{1}$ Department of Pediatrics, First Affiliated Hospital of Anhui Medical University, Hefei, China \\ ${ }^{2}$ Department of Medical Technology, Anhui Medical College, Hefei, China \\ Email: yuanliping3986@sina.com
}

Received 7 December 2013; revised 5 January 2014; accepted 13 January 2014

Copyright (C) 2014 by authors and Scientific Research Publishing Inc.

This work is licensed under the Creative Commons Attribution International License (CC BY). http://creativecommons.org/licenses/by/4.0/

(c) ()

\section{Abstract}

Background: Henoch-Schönlein purpura (HSP) is a kind of systemic small vessel vasculitis in children. Endothelium cells injury induced by IgA1 is considered important in the pathogenesis of HSP. Research found that the apoptosis of vein endothelial cells was related to the vasculitis in HSP patients. Purpose: To observe the effect of IgA1 from HSP patients on the apoptosis of HUVEC and firstly analyze the mechanism of the apoptosis of HUVEC induced by IgA1. Methods: HUVECs were cultured in 3 different conditional media with IgA1 from HSP patients, normal healthy children and simply medium (blank control). Serum IgA1 was purified by jacalin affinity chromatography. The rates of apoptosis in HUVEC incubated with IgA1 were determined by TUNEL method and flow cytometry, respectively. The expression of the cytoskeletal proteins, such as FAK, Vinculin and MLCK was detected with the methods of Real-time PCR and Westernblot, respectively. Results: The present study showed that the apoptosis rate of HUVEC by IgA1 isolated from HSP patients was higher than blank control $(14.77 \% \pm 2.23 \%$ vs $2.25 \% \pm 0.77 \%)(P<0.01)$ and the rate of HUVEC by IgA1 from normal healthy children was higher than blank control $(9.97 \% \pm 1.48 \%$ vs $2.25 \% \pm 0.77 \%)(P<0.01)$. The cytoskeletal proteins, such as FAK, Vinculin and MLCK expression were down-regulated in HUVEC co-cultured with IgA1 isolated from HSP patients for 24h. Conclusion: These findings firstly on IgA1 from HSP patients may induce apoptosis of vascular endothelial cells through inhibiting the cytoskeletal proteins expression. IgA1 may accelerate progression of HSP by inducing apoptosis of vascular endothelial cells.

\section{Keywords}

Henoch-Schönlein Purpura (HSP); Endothelial Cells; Immunoglobulin A; Apoptosis; Cytoskeletal Proteins

${ }^{*}$ Corresponding author. 


\section{Introduction}

Henoch-Schönlein purpura (HSP) is the most common systemic vasculitis that occurs commonly in children. It is characterized by a systemic leukocytoclastic angiitis, mainly affecting the small vessels of the skin, joints, gastrointestinal tract, and kidneys. Other organs, such as brain, lungs, and scrotum, may occasionally be involved. Though the cause has not been well elucidated, it is confirmed that widespread leukocytoclastic vasculitis caused by IgA, especially IgA1 deposition in the small vessel walls played an important role in the pathogenesis of HSP [1]. However, the aetiology and pathogenesis of HSP are still fully unknown. Recent research has showed that the supernatant of peripheral blood mononuclear cells co-cultured with serum from HSP patients could induce the apoptosis of human umbilical endothelial cells (HUVEC) [2]. Later our study also found the apoptosis of vein endothelial cells (VEC) in skin tissue of HSP patients, which was correlated with high IgA level in serum [3]. These results suggested that the apoptosis of VEC may play an important role in the pathogenesis of HSP. To further investigate the apoptosis in vein endothelial cells of HSP patients and its correlation with IgA1, we isolated IgA1 from sera at the acute stage of childhood HSP to firstly analyze the apoptosis of HUVEC induced by IgA1and its mechanism in the present study.

\section{Methodology}

10 HSP patients (6 boys, 4 girls; range, 6 - 14 years) admitted to our hospital, were selected according to the EULAR/PReS endorsed consensus 2005 criteria [4]. Active sera were isolated from whole blood for isolation of circulating IgA1 at the acute stage and before steroid or other immunosuppressants treatment. Ten age-matched healthy children were also enrolled as normal healthy controls. Informed consents were obtained, and the study was approved by hospital institutional review boards.

The circulating IgA1 was isolated from sera by Immobilized Jacalin (Pierce, Rockford, IL, USA) and the level of IgA1 was assayed by ELISA methods.

Human umbilical vein endothelial cells (HUVECs) were isolated following standard protocols. The cells were treated with isolated IgA1 (200 ug/ml) at $37^{\circ} \mathrm{C}$ for $24 \mathrm{~h}$ from HSP patients and normal healthy children, respectively. The blank control cells were not treated with either IgA1. The apoptosis of HUVEC was detected using flow cytometry and TUNEL (Roche, Germany) according to the supplier's instructions, and apoptosis index (AI) was used to evaluate cell apoptosis.

Real-time quantitative polymerase chain reaction (PCR) was performed to determine on RNA expression for FAK, Vinculin and MLCK. Total RNA were isolated from HUVEC cells using Trizol reagent (Invitrogen, Shanghai, China). Total RNA ( $1 \mu \mathrm{g})$ was reverse transcribed into cDNA using AMV reverse transcriptase (Fermentas,). The primers for FAK, Vinculin, MLCK and $\beta$-actin were as follows (Table 1). Conditions for the PCR were as follows: $95^{\circ} \mathrm{C}$ for 4 minutes, followed by 35 cycles for FAK, Vinculin and MLCK or 30 cycles for $\beta$-actin. The PCR products were run on an agarose gel and were in all cases confined to a single band of the expected size. $2^{-\triangle \mathrm{CT}}$ was used to figure the expression value of FAK, Vinculin and MLCK.

FAK, Vinculin and MLCK protein was analyzed in whole cell HUVEC lysates $(50 \mu \mathrm{g} / \mathrm{lane})$ using a commercially available antibody (Abcam) as described [5].

\section{Statistical Analyze}

Statistical comparisons of mean values were performed using Student's t-test and Kruskal-Wallis Test of SPSS 13.0 software. All P-values were determined from two-sided tests. A significance criterion of $\mathrm{P}<0.05$ was used in these studies.

Table 1. Primer pares.

\begin{tabular}{|c|c|c|c|}
\hline \multirow{2}{*}{ Gene } & \multicolumn{2}{|c|}{ Primer sequence (5'-3') } & \multirow{2}{*}{ Products size (bp) } \\
\hline & Forward & Reverse & \\
\hline vinculin & CCAGCAAGCCGGGCATCCCA & CCCGCTTGGTACCACTGCCC & 303 \\
\hline MLCK & AGGAGATCCCGGCCGAGCAG & TCTCGGCAGGCTTGGCGTTG & 390 \\
\hline FAK & GACTGCTGCTCACGCCCTGG & TAGCTCTCСTCTTCCACTATGCAGC & 489 \\
\hline actin & AAGAGATGGCCACGGCTGCT & GACTCGTCATACTCCTGCTTGCT & 421 \\
\hline
\end{tabular}




\section{Results}

\subsection{Apoptosis of HUVEC Induced by IgA1 from HSP Patients}

In the study we firstly examined the apoptosis rate of HUVEC using flow cytometry and TUNEL methods, respectively. Flow cytometry demonstrated that apoptosis rate of HUVEC by IgA1 $(200 \mu \mathrm{g} / \mathrm{ml})$ isolated from HSP patients was higher than blank control $[(14.77 \pm 2.23) \%$ vs. $(2.25 \pm 0.77) \%, \mathrm{P}<0.01]$ and the rate of HUVEC by IgA1 from normal healthy children was higher than blank control [(7.97 \pm 1.48$) \%$ vs. $(2.25 \pm 0.77) \%$, P < 0.01]. Moreover IgA1 $(200 \mu \mathrm{g} / \mathrm{ml})$ from HSP patients markedly induced the apoptosis death of HUVEC compared with normal control group $(\mathrm{P}<0.01)$ (Table 2). Similar results were observed using TUNEL methods (Table 2).

\subsection{Effect of IgA1 from HSP Patients on the Expression of FAK, Vinculin and MLCK in HUVEC}

In addition, we preliminary explored the FAK, Vinculin and MLCK in HUVEC induced by IgA1. PCR showed that IgA1 isolated from HSP patients could significantly decrease the mRNA expression of cytoskeletal proteins, FAK, Vinculin and MLCK for $24 \mathrm{~h}$. There had an obvious difference between HSP group and blank control group, normal control group $(\mathrm{P}<0.01, \mathrm{P}<0.05)$ (Table 3). Similar results were observed using Western blot method (Table 3 and Figure 1).

\section{Discussion}

This study demonstrated induction by isolated IgA1 from HSP patients of apoptosis in human vascular endothelial cells. Flow cytometry and TUNEL results showed that IgA1 isolated from HSP patients could significantly increase the apoptosis rates of HUVEC compared with IgA1 isolated from normal healthy children and without IgA1.

It was estimated that IgA1-mediated endothelium injury played a key role in the pathogenesis of HSP [6] [7]. Endothelium is a highly dynamic tissue that controls vascular homeostasis. This requires constant rearrangements of the shape or function of endothelial cells that cannot set aside the role of the cytoskeleton. It has been accepted that apoptosis in endothelial cells led to the rearrangements of the shape and endothelial barrier dysfunction. Mounting evidence implicates cytoskeleton proteins, as a sensor and mediator of apoptosis, significantly contribute to apoptosis and play a key role in regulating apoptosis [8]. Focal adhesion kinase (FAK), a prominent component of cytoskeleton proteins, is critically positioned to integrate signals from the extracellular

Table 2. Effect of IgA1 from different groups on apoptosis rate of HUVEC $(\mathrm{X} \pm \mathrm{S})$.

\begin{tabular}{cccc}
\hline Group & $\mathrm{n}$ & Apoptosis rate \% (FCM) & AI \% (TUNEL) \\
\hline Blank control & 8 & $2.25 \pm 0.77$ & $2.17 \pm 0.49$ \\
HSP & 8 & $14.77 \pm 2.23^{* * \boldsymbol{\Lambda}}$ & $13.64 \pm 2.95^{* * \boldsymbol{\Lambda}}$ \\
Normal control & 6 & $9.97 \pm 1.48^{* *}$ & $6.32 \pm 1.41^{* *}$ \\
\hline
\end{tabular}

${ }^{* *} \mathrm{P}<0.01$, compared with blank control; ${ }^{\mathbf{\Lambda} \Lambda} \mathrm{P}<0.01$, compared with normal control.

\section{Table 3. Expression of FAK, paxillin and vinculin mRNA in HUVEC $(\mathrm{X} \pm \mathrm{S})$.}

\begin{tabular}{|c|c|c|c|c|c|c|c|}
\hline \multirow{2}{*}{ Group } & \multirow{2}{*}{$\mathrm{n}$} & \multicolumn{3}{|c|}{ mRNA expression } & \multicolumn{3}{|c|}{ Protein expression } \\
\hline & & vinculin & MLCK & FAK & vinculin & MLCK & FAK \\
\hline $\begin{array}{c}\text { Blank } \\
\text { control }\end{array}$ & 8 & $0.40 \pm 0.02$ & $0.55 \pm 0.01$ & $0.42 \pm 0.02$ & $0.35 \pm 0.02$ & $0.41 \pm 0.03$ & $0.53 \pm 0.04$ \\
\hline HSP & 8 & $0.13 \pm 0.01^{* * \Delta \Delta}$ & $0.21 \pm 0.03^{* * \boldsymbol{\Lambda}}$ & $0.12 \pm 0.01^{* * \Delta \Lambda}$ & $0.16 \pm 0.03^{* * \boldsymbol{\Lambda}}$ & $0.18 \pm 0.01^{* * \Delta \Lambda}$ & $0.26 \pm 0.02^{* * \boldsymbol{\Lambda}}$ \\
\hline $\begin{array}{l}\text { Normal } \\
\text { control }\end{array}$ & 6 & $0.28 \pm 0.01^{*}$ & $0.35 \pm 0.01^{*}$ & $0.26 \pm 0.03$ & $0.20 \pm 0.01^{*}$ & $0.25 \pm 0.02^{*}$ & $0.37 \pm 0.03$ \\
\hline
\end{tabular}

${ }^{*} \mathrm{P}<0.05,{ }^{* *} \mathrm{P}<0.01$, compared with blank control; ${ }^{\boldsymbol{\Delta}} \mathrm{P}<0.05,{ }^{\boldsymbol{\Delta}} \boldsymbol{\Delta} \mathrm{P}<0.01$, compared with normal control. 


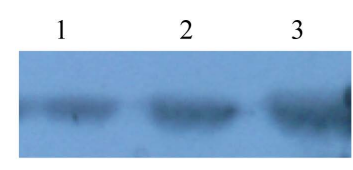

vinculin

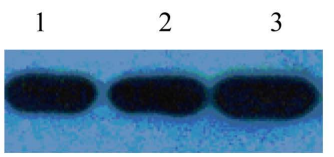

$\beta$-actin

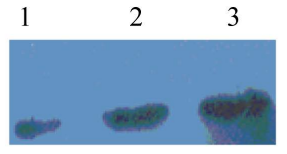

MLCK

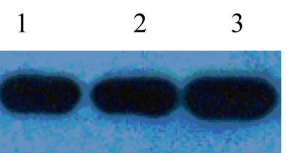

$\beta$-actin

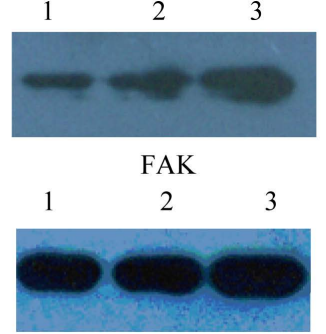

$\beta$-actin

Figure 1. FAK, vinculin and MLCK protein expression in HUVEC by Westernblot method. 1. HSP group; 2. normal control group; 3. blank control group.

matrix and cellular adhesion. It is essential for normal vascular development and has been implicated in a wide range of cellular functions including the regulation of cell proliferation, migration, differentiation, and survival. Resaerches showed the loss of epithelial FAK resulted in elevated p53 expression and an increased sensitivity to apoptosis, coincident with a failure to upregulate epithelial cell proliferation [9]. FAK has been reported to function as a mechanosensor, inducing cyclin D1 expression and promoting cell cycle progression under conditions in which tissue/matrix stiffness is increased [9]. Vinculin, another cytoskeleton protein, is a cytoplasmic actin-binding protein enriched in focal adhesions and adherens junctions that is essential for embryonic development. The recent data suggests a critical function for vinculin in regulating integrin clustering, force generation, and strength of adhesion [10]. In addition to an important role in cell-matrix adhesion, vinculin is also emerging as a regulator of apoptosis. Our present study presented that IgA1 from HSP patients could downregulate the expression of vinculin and FAK in HUVEC mRNA and protein comapred with IgA1 isolated from normal healthy children and without IgA1. This result showed that IgA1 may mediate endothelium apoptosis by inhibiting the cytoskeleton proteins.

Myosin Light chain kinases (MLCK) are $\mathrm{Ca}^{2+} /$ calmodulin-activated enzymes that trigger actomyosin interaction and initiate contraction in smooth muscle cells [11]. Phosphorylation of MLCK regulates diverse cellular functions involving the interaction of myosin and actin at the level of the cytoskeleton. The research showed that MLCK plays a role in cell migration, cell retraction, cell barrier regulation, transendothelial migration of neutrophils, and apoptosis [12]. In this experiment we found that the MLCK expression of HUVEC was decreased by IgA1 from HSP comapred with IgA1 isolated from normal healthy children and without IgA1.

\section{Conclusion}

In summary, IgA1 from HSP patients was firstly found to induce apoptosis of vascular endothelial cells, which may be related to inhibiting the cytoskeletal proteins expression. Moreover, IgA1 may accelerate progression of HSP by inducing apoptosis of vascular endothelial cells.

\section{Acknowledgements}

This research was supported by the Project supported by the National Natural Science Foundation of China (NO 81001339).

\section{References}

[1] Ballinger, S. (2003) Henoch-Schonlein Purpura. Current Opinion in Rheumatology, 15, 591-594. http://dx.doi.org/10.1097/00002281-200309000-00012

[2] Le, X., Zeng, C.H., Bao, Z.X., He, Z.Q. and Li, X.D. (2005) Effect of Inflammatory Cytokines on the Vascular Endothelial Cells Injured of HSP Patients and Its Mechanism. Guang Dong Medicine, 26, 520-521. (In Chinese)

[3] Yuan, L.P., Zhang, Q. and Lu, L. (2012) Apoptosis of Vascular Endothelial Cells from HSP Patients and Its Relation with Serum IgA. Chinese Journal of Immunology, 28, 81-83. (In Chinese)

[4] Ozen, S., Ruperto, N., Dillon, M.J., Bagga, A., Barron, K., et al. (2006) EULAR/PReS Endorsed Consensus Criteria for the Classification of Childhood Vasculitides. Annals of the Rheumatic Diseases, 65, 936-941. 
http://dx.doi.org/10.1136/ard.2005.046300

[5] Fukuda, R., et al., (2007) HIF-1 Regulates Cytochrome Oxidase Subunits to Optimize Efficiency of Respiration in Hypoxic Cells. Cell, 129, 111-122. http://dx.doi.org/10.1016/j.cell.2007.01.047

[6] Yang, Y.H., Huang, Y.H., Lin, Y.L., Wang, L.C., Chuang, Y.H., et al. (2006) Circulating IgA from Acute Stage of Childhood Henoch-Schönlein Purpura Can Enhance Endothelial Interleukin (IL)-8 Production through MEK/ERK Signalling Pathway. Clinical \& Experimental Immunology, 144, 247-253. http://dx.doi.org/10.1111/j.1365-2249.2006.03076.x

[7] Zhang, Q., Lu, L. and Lin, D. (2008) Levels and Significance of IgA1 in Serum and Skin Lesions of Pediatric Patients with Anaphylactoid Purpura. Chinese Journal of Dermatology, 41, 55-56. (In Chinese)

[8] Desouza, M., Gunning, P.W. and Stehn, J.R. (2012) The Actin Cytoskeleton as a Sensor and Mediator of Apoptosis. Bioarchitecture, 2, 75-87. http://dx.doi.org/10.4161/bioa.20975

[9] Owen, K.A., Abshire, M.Y., Tilghman, R.W., Casanova, J.E. and Bouton, A.H. (2011) FAK Regulates Intestinal Epithelial Cell Survival and Proliferation during Mucosal Wound Healing. PLoS One, 6, e23123. http://dx.doi.org/10.1371/journal.pone.0023123

[10] Peng, X., Nelson, E.S., Maiers, J.L. and DeMali, K.A. (2011) New Insights into Vinculin Function and Regulation. International Review of Cell and Molecular Biology, 287, 191-231. http://dx.doi.org/10.1016/B978-0-12-386043-9.00005-0

[11] Wang, H.H., Nakamura, A., Yoshiyama, S., Ishikawa, R., Cai, N., Ye, L.H., Takano-Ohmuro, H. and Kohama, K. (2012) Down-Regulation of Myosin Light Chain Kinase Expression in Vascular Smooth Muscle Cells Accelerates Cell Proliferation: Requirement of Its Actin-Binding Domain for Reversion to Normal Rates. Journal of Pharmacological Sciences, 119, 91-96. http://dx.doi.org/10.1254/jphs.11213SC

[12] Woodfin, A., Voisin, M.B., Beyrau, M., Colom, B., Caille, D., Diapouli, F.M., Nash, G.B., Chavakis, T., Albelda, S.M., Rainger, G.E., Meda, P., Imhof, B.A. and Nourshargh S. (2011) The Junctional Adhesion Molecule JAM-C Regulates Polarized Transendothelial Migration of Neutrophils in Vivo. Nature Immunology, 12, 761-769. http://dx.doi.org/10.1038/ni.2062 